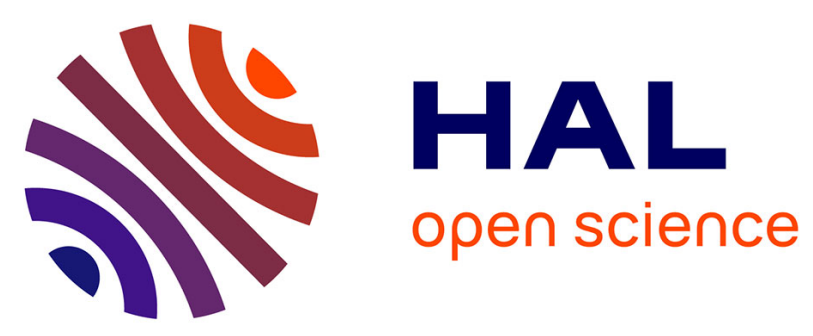

\title{
La céramique argonnaise du IVe siècle décorée à la molette à Guiry-Gadancourt (Seine-et-Oise)
}

Pierre-Henri Mitard

\section{To cite this version:}

Pierre-Henri Mitard. La céramique argonnaise du IVe siècle décorée à la molette à Guiry-Gadancourt (Seine-et-Oise). Gallia - Fouilles et monuments archéologiques en France métropolitaine, 1958, 16 (2), pp.293-299. 10.3406/galia.1958.2239 . hal-01924464

\section{HAL Id: hal-01924464 \\ https://hal.science/hal-01924464}

Submitted on 3 Mar 2020

HAL is a multi-disciplinary open access archive for the deposit and dissemination of scientific research documents, whether they are published or not. The documents may come from teaching and research institutions in France or abroad, or from public or private research centers.
L'archive ouverte pluridisciplinaire HAL, est destinée au dépôt et à la diffusion de documents scientifiques de niveau recherche, publiés ou non, émanant des établissements d'enseignement et de recherche français ou étrangers, des laboratoires publics ou privés.

\section{(ㅇ)(1) $\$$}

Distributed under a Creative Commons Attribution - NonCommercial - NoDerivatives| 4.0 


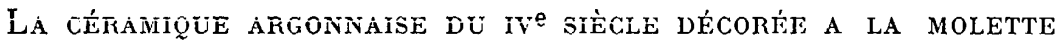 a Guiry-Gadancourt (Seine-rit-Oise)}

La céramique argonnaise du Iv $^{\mathbf{e}}$ siècle présente pour le fouilleur l'avantage de ne pouvoir être confondue avec aucune autre, puisque - du moins jusqu'à preuve du contraire - les ateliers d'Argonne sont les seuls à avoir produit ce type de céramique, caractérisée par une coloration rouge-orangé clair et un décor imprimé à la molette. En rendant aisée l'identification, cet élément compense ce que peut avoir d'ingrat l'étude de cette céramique, comparée à celle de la sigillée des I $^{\text {er }}$ II $^{\mathrm{e}}$ siècles, dont elle est cependant l'héritière ou dont elle constitue la survivance. La publication du bel ouvrage de G. Chenet ${ }^{1}$ ayant maintenant appelé largement l'attention sur cette céramique, il semble que chaque fouilleur devrait avoir à cœur d'apporter sa contribution à la connaissance de celle-ci par la publication de ses propres trouvailles dans ce domaine. C'est dans cet esprit que nous avons rédigé cette note consacrée à la céramique argonnaise du Ive siècle recueillie au cours de la première campagne de fouille de la villa gallo-romaine des "Terres Noires" à Guiry-Gadancourt (Seine-et-Oise) ${ }^{2}$.

Outre les fragments de vases décorés à la molette, la récolte comprend des vases unis - trouvés en morceaux, mais dont la reconstitution a été possible ainsi que des tessons - qui, à dẹfaut, de reconstitution, permettent de reconnaitre

(1) G. CrIEnet, La céramique gallo-romaine d'Argonne du $I V^{\mathrm{c}}$ siècle el la terre sigillèe décorée à la moletle, Måcon, 1941.

(2) Voir le compte rendu de cette fouille dans le présent tome de Gallia, plus haut; les numéros de zones indiqués plus loin renvoient au plan qui illustre cette Note (fig. 1). la forme des vases auxquels ils appartenaient - attribuables également, par la couleur et la forme, aux officines argonnaises. Nous commencerons par donner quelques indications à leur sujet.

Vases el lessons de vases non décorés à la molette: les vases ont été recueillis dans la salle 4, les tessons dans la fosse 24.

1. $\left.(0.290)^{3} .1\right)=28$ centimètres (fig. 1 , $n^{0}$ 1) : plat creux, à pied évidé, à marli horizontal. Type Chenet 313, avec marli très proche de celui représenté dans l'ouvrage de référence, fig. $18, n^{0} 7$. Engobe de qualité, bien conservé, mais certains fragments ont été noircis par le feu alors que le plat étail déjà brisé.

2. (0.343). Hauteur probable : 25 centimètres, manque le fond (fig. 1, no 2) : cruche globuleuse à col allongé conique, à orifice circulaire à bec pincé avec anse plate à nervure. Type Chenet 348 , terre rose à engobe rouge-orangé clair, mal conservé.

3. $(0.522)$. D. $=20$ centimètres environ (fig. $1, n^{\circ} 3$ ) : écuelle hémisphérique à marli horizonlal lisse, à pied cylindrique évidé. Type Chenet $314 \mathrm{~b}$, var. (marli un peu différent). Le vase est très incomplet, l'engobe est devenu brun sous l'action du feu, qui a fait éclater la terre.

4. (0. 460). (D. $=16$ centimètres) (fig. 1 $\mathrm{n}^{0}$ 4) : terrine à large bord vertical à baguettes, lisse, à pied conique et à intérieur lisse. Type Chenet $324 \mathrm{c}$, var.

(3) Les numéros précédés de la lettre $O$ sont ceux sous lesquels les objets sont enregistrés au Musée de Guiry. 

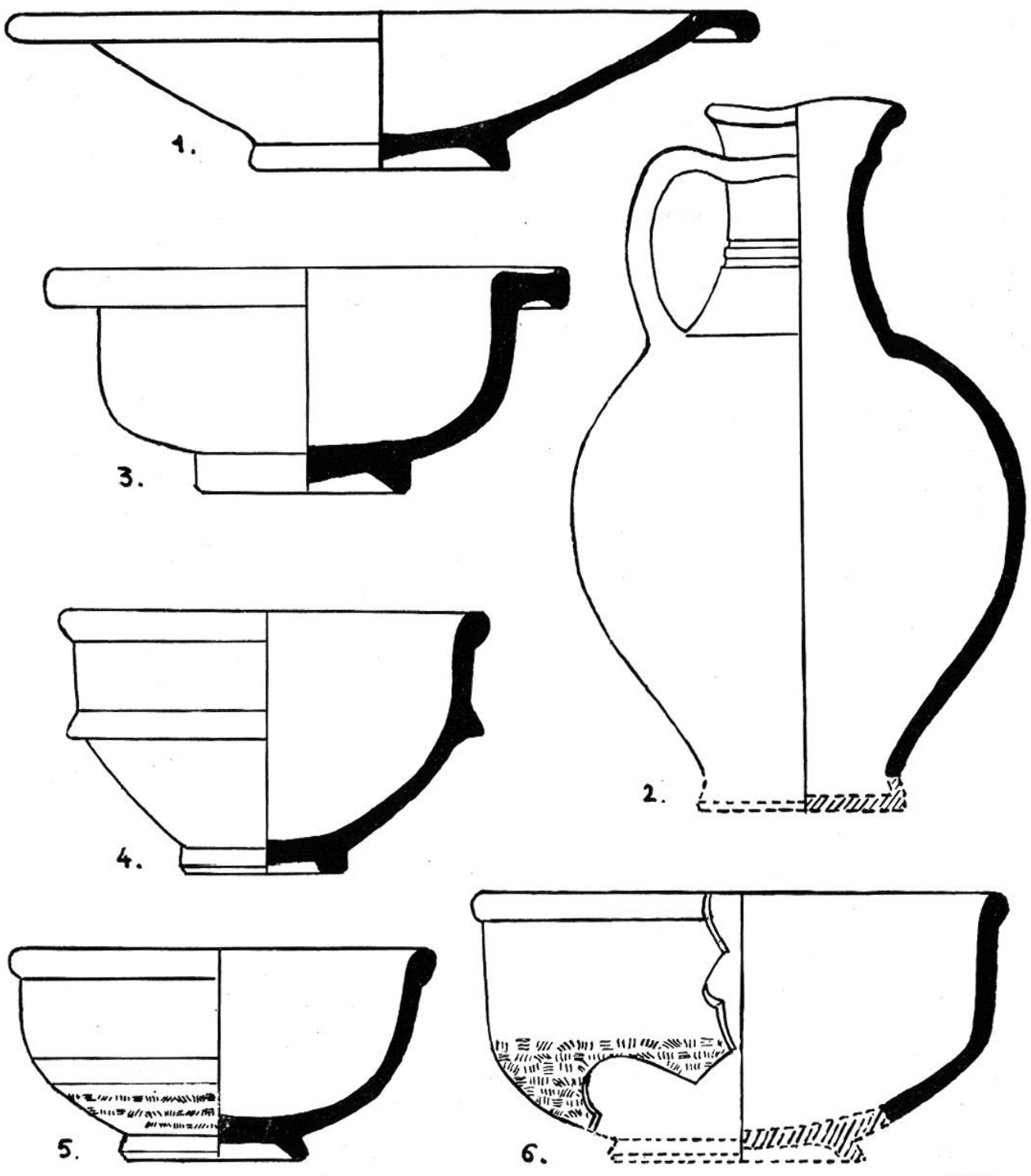

Fig. 1. - Vases argonnais du Iv siècle. Échelle : 1/3.

B.H.

Engobe rouge-orangé clair très mal conservé, vase ayant subi le feu après brisure.

5. Fragments divers : fragment de bord de terrine à déversoir en forme de mufle de lion d'un style barbare, type Chenet 330 , terre rose à peine vernissée sauf au sommet du bord; pour le reste, aspect plus proche de la terre commune que de la sigillée; - fragments divers, dont certains, portant un décor à la barbotine, proviennent d'un gobelet ovoïde à col profilé du type Chenet 334, terre rose à engobe rouge-orangé clair d'excellente qualité (diamètre extérieur du col : 5 centimètres, épaisseur de la paroi : 
2 millimètres); un autre col d'un vase du même type, provenant de la même zone, a 7 centimètres de diamètre; fragment de bord de tasse conique, type Chenet 310 (diamètre $=8 \mathrm{~cm}$. 5) ; fragment de bord de tasse hémisphérique, type Chenet 319 a ; - fragment de bord de plat ou jatte à bord relevé, type Chenet 304.

On a recueilli en outre, principalement dans la fosse 24, plus d'une centaine de tessons de céramique à vernis rouge orangé clair. Certains de ceux-ci appartenaient à plusieurs vases à intérieur garni de quartz, les autres probablement en majorité à des bols du type Chenet 320 tels que ceux qui sont étudiés ci-après.

Céramique décorée à la molelte : elle est représentée par un bol (très incomplet) et 52 tessons indépendants (non recollables entre eux). Ces tessons (numérotés Ar. 1 à 16 el Ar. 18 à 41) portent des décors issus de 40 molettes différentes, auxquelles il faut ajouter celle du bol précité $(0.487)$. Ils appartenaient, outre ce bol, à 42 au moins, peut-être 44 , vases différents; c'est-à-dire que, en deux cas au moins - et peut être quatre - la même molette se retrouve sur des tessons appartenant à deux vases différents. Quatorze seulement de ces tessons (Ar. 5 a et b, 6, $91011,15,18,25$ a, $26,37,38,40$ et 41 ), ainsi que le bol, ont été trouvés à l'intérieur ou aux abords immédiats de celui-ci (le bol, salle 4). Tous les autres tessons, sous réserve de quelques trouvailles de surface, proviennent des fosses-dépotoirs 13 et surtout 24.

A. Forme et matière. - Tous les tessons portant un décor à la molette appartiennent à des bols des diverses variétés de la forme Chenet 320 ; ccux de ces tessons qui sont les moins caractéristiques ne peuvent en effet, compte tenu des formes connues pour associées au décor à la molette, appartenir à des vases d'un autre type. Les dimensions de ces bols ne peuvent être déterminées que dans un petit nombre de cas : pour 0. 487 (fig. 1, no 5), l'ouverture à un diamètre de 14 centimètres et le pied de $7 \mathrm{~cm}$. 2, la forme est assez lourde ; pour Ar. 1 (reconstitution graphique fig. $1, n^{0} 6$ ), l'ouverture avait un diamètre de 18 centimètres et la forme est plus légère. Citons encore à titre indicatif Ar. 36 : 19 centimètres d'ouverture, $\Lambda$ r. $20: 8$ millimètres de diamètre au pied. L'épaisseur des parois va de 4 à 8 millimètres, le plus grand nombre de tessons (plus de la moitié) ayant des épaisseurs comprises entre $4 \mathrm{~mm}$. 5 et $6 \mathrm{~mm}$. 5. Ces indications peuvent ctre complétées par celles résultant des observations faites sur des tessons non décorés eux-mêmes (bords), mais appartenant certainement à des bols Chenet 320 , décorés à la partie inféricure. Sur la quarantaine de bords de bols différents recueillis, une vingtaine permettent de déterminer le diamètre de l'ouverture des bols correspondants : celui-ci allait de 11 à 24 centimètres, les plus nombreux se tenant à 14 et 15 centimètres (respectivement 8 et 4 pièces).

L'engobe est d'unc manière générale de la teinte rouge-orangé clair, classique pour cette céramique; il est de bonne qualité (bien conservé) dans la moitié des cas; la pâte elle-même est d'une teinte un peu plus claire. Dans trois cas $(\Lambda$ r. 3 , 8 el 23) la teinte de l'engobe est plus brune probablement par suile d'une surcuisson. I'un de ces tessons plus bruns (Ar. 23) est décoré d'une molette à damier (cf. infra, variantes de molettes déjà connues), ce qui rejoint une observation faite par M. Gricourt à Bavai ${ }^{4}$,

(4) Jean Gricourt, La terre sigillée argonnaise du Ive siècle décorée à la molette à Bavai (Nord), Gallia, VIII, 1950, cf. p. 61, n. 14. 
selon laquelle la plupart des tessons décorés d'une telle molette sont bruns (ou ont bruni) et souvent détériorés. Le bol (0. 487) présente une terre grisâtre et un engobe franchement brun qui doivent au contraire être attribués à l'incendie. A l'opposé, la terre de deux tessons est d'un rose proche du blanc; cependant, tandis que sur l'un ( $\mathrm{Nr} .13$ ), l'engobe a presque complètement disparu, sur l'autre (Ar. 16), il est intact et de bonne qualité.

B. Les molettes. - Sur les 41 molettes observées, 4 seulement sont à considérer avec certitude comme déjà connues, auxquelles on peut ajouter trois autres dont l'identification est moins certaine (en raison de l'exiguïté des tessons); 10 peuvent être des variantes de molettes connues; 16 sont inédites; 8 enfin sont probablement nouvelles également mais, en raison du mauvais état ou de l'exiguité des tessons qui les portent, nous ne pouvons que les classer comme incertaines.

On remarque le nombre très faible de molettes déjà connues, de l'ordre de 10 à $15 \%$ au total. Quant aux inédites, nous les considérons comme telles en tenant compte, outre les 354 figurant dans l'ouvrage de base de G. Chenet, des 37 trouvées au cours des fouilles récentes de Bavai et publiées par M. J. Gricourt ${ }^{5}$.

Restent les "variantes " de molettes connues. M. Gricourt se demandait à l'occasion de son étude : "comment tracer une démarcation entre les simples variantes et les molettes inédites ?" et ajoutait : "A ce problème, il ne peut y avoir de solution que personnelle". Nous en sommes bien d'accord, mais sous réserve de l'observation suivante : le problème n'existe qu'en raison de la

(5) Op. cit. difficulté que l'on rencontre pour représenter chaque molette avec une fidélité parfaite. La démarcation nous paraît donc résider dans la possibilité ou la non possibilité de distinguer avec certitude, d'après une représentation graphique, la molette en cause de celles déjà publiées, ou - si l'on se place d'un autre point de vue - dans la possibilité, qu'aurait lc lecteur, de distinguer avec certitude les deux molettes d'après leurs représentations graphiques, compte tenu de l'inévitable imperfection des deux représentations. Si l'on n'a pas cette certitude, ou du moins si l'on ne croit pas pouvoir la faire partager à ses lecteurs, si les différences constatées risquent d'être imputées à une fidélité insuffisante de la représentation, il vaut mieux considérer la molette comme variante de telle molette déjà connue. Mais dans le cas contraire, même si les différences sont minimes, pourquoi ne pas considérer la molette comme "inédite » et la publier comme telle? Deux molettes peuvent être de types très proches; si elles sont distinctes, elles pcuvent, étant donné en particulier le caractìre simple des motifs qui facilite les imilations et même les rencontres, avoir appartenu à des ateliers ou des dates différents et justifier par conséquent des études différenciées qu'il importe de permeltre en publiant la seconde. Telles sont les considérations dont nous nous sommes inspirés dans les cas litigicux.

Les molettes inédites sont représentées à la figure 2 et la nomenclature en est donnée ci-après. $\Lambda$ celles provenant du chantier faisant l'objet du présent rapport nous avons ajouté une molette (no 17) figurée sur un tesson ramassé en surface à un autre emplacement : au lieudit "Les Étots", à 1 kilomètre au NordOuest de ce chantier. Les tessons en 
1

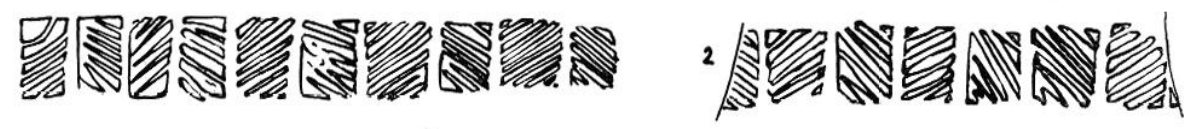

3

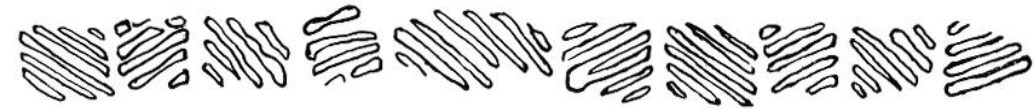

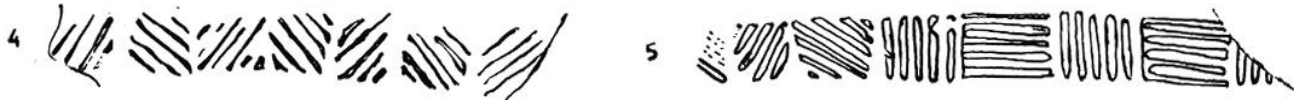

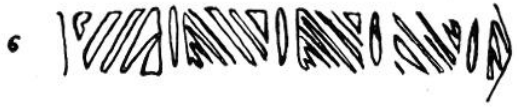

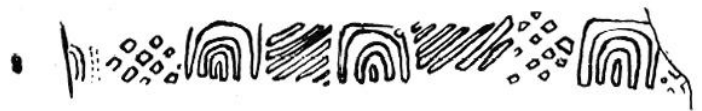

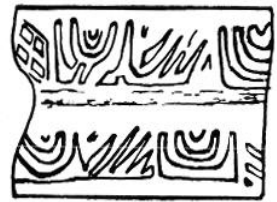

10

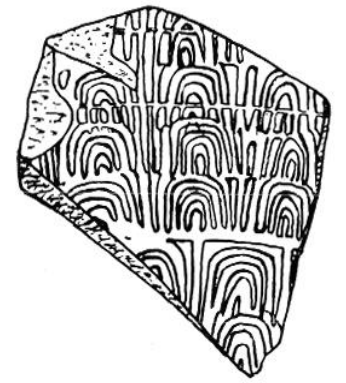

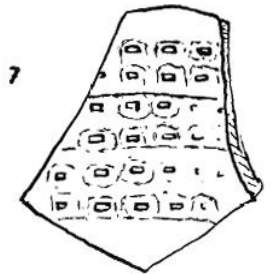

11

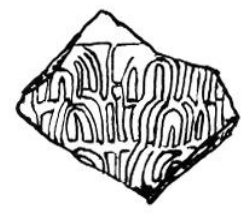

12 *

13

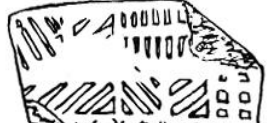

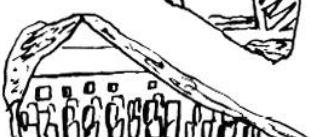

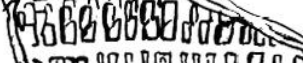

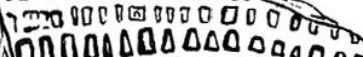

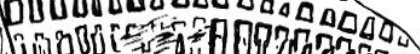

16

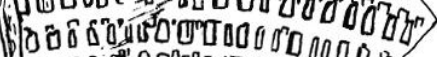

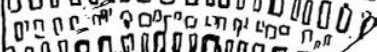

$300000,0,4500000000^{2}$

8010100000000

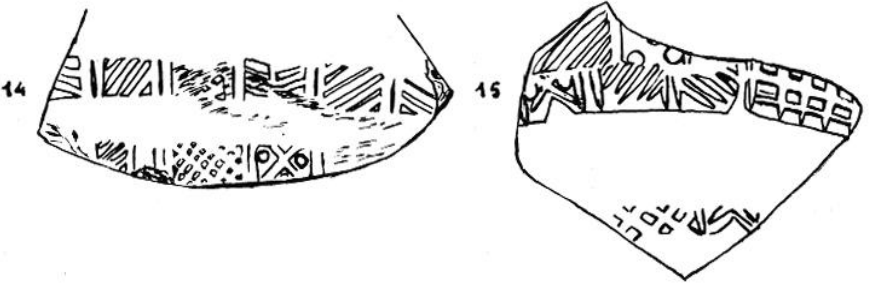

17

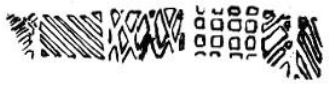

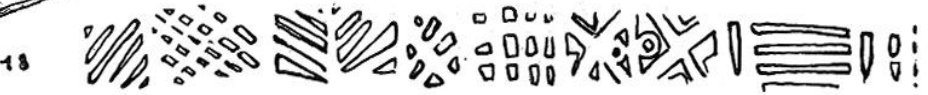

Fig. 2. - Nos 1 à 17 : molettes inédites trouvées à Guiry-Gadancourt. No $18:$ molette 212 de Unverzagt et Chenet, complétée par un relevé sur un vase de Guiry-Gadancourt. Grandeur naturelle. Relevé de P.-H. Mitard. 
cause portent les numéros Ar. 1 à 17, correspondant à ceux de la nomenclature.

1. Molette complète : 10 casiers ; caractérisée par la finesse des reliefs et leur faible accentuation; sur 5 tessons indépendants paraissant appartenir à 2 vases distincts;

2. Incomplète : au moins 7 casiers; sur 5 tessons indépendants appartenant à au moins 2 vases différents;

3. Complète : 10 casiers; dont un double des autres en longucur;

4. Incomplète : au moins 7 casiers, facture grossière, creux très étroils;

5. Incomplète : au moins 8 casicrs, à rapprocher des mol. 23 à 31 de G. Chenet (d'après Unver\%agt), différentes cependant par l'alternance des divers casiers et les intervalles entre les casiers à hachures obliques ${ }^{6}$;

6. Incomplète : au moins 5 casiers;

7. Incomplète : deux bandes de creux étroits au centre de dépressions peu accentuées ;

8. Incomplète (?) : au moins 7 casiers, le casier incertain figuré à gauche peut être le même que celui de motif similaire figuré à droite ; dans ce cas, la molette serait complète et à 7 casiers; à rapprocher de mol. 140 de G. Chenet (d'après Unverzagt) ;

9. Incomplète : au moins 8 casiers, à rapprocher également de mol. $140 \mathrm{de}$ G. Chenet, bien que d'une exćcution différente; le tesson représenté est fictif : la $1^{\text {re }}$ bande a été complétée par un report à partir d'une bande située au-dessous de la $2^{e}$;

(6) Une plus grande familiarité avec les molettes d'Argonne nous permet maintenant de penser que cette molette pourrait être celle du no 26 de G. Chenet, d'après la représentation, beaucoup trop schématique, de W. UnverzaGt.
10. Incomplète : au moins 6 casiers; le chevauchement des bandes crée un effet décoratif particulier, mais rend difficile la reconstitution de la molette ; le $1^{\mathrm{er}}$ casier de la $2^{\mathrm{e}}$ bande est la même que le dernier des $3^{\mathrm{e}}$ et $4^{\mathrm{e}}$; à rapprocher des mol. 142,145 et 146 , mais les oves sont ici à centre creux (bandes inférieures) ou à 4 élćments à centre plein, au lieu de 3 éléments ct centre plein;

11. Incomplète : oves très grêles;

12. Incomplète : au moins 11 casiers; sur tesson de pâte et engobe d'une particulièrement bonne qualité ;

13. Incomplète : au moins 9 casiers : le casier lisible de la bande inférieure peut être le même que le 1 er de la $2^{2}$ bande; à rapprocher de mol. 148 de G. Chenet (d'après Unverzagt) ;

14. Incomplète : au moins 9 casiers ;

15. Incomplète : au moins 7 casiers : le dernier casier de la bande inférieure est très probablement - un détail l'indique malgré la déformation de l'empreinte - le même que le $1^{\text {er }}$ de la $2^{\mathrm{e}}$ bande (une bande intermédiaire qui faisait double emploi n'a pas été représentée) ; à rapprocher de mol. 123 de G. Chenet (d'après Unverzagt);

16. Incomplète : deux bandes de creux de largeur variable à rapprocher de mol. 158 de G. Chenet (d'après Unverzagt), mais à l'inverse de cette dernière les creux sont ici plus hauts que larges; à rapprocher surtout de la molette décorant le vase qui figure sur la deuxième des planches photographiques insérées après la page XII de l'ouvrage de G. Chenet (photo en haut à droite - les deux vases paraissent également avoir été très proches de forme et de dimension);

17. Incomplète : au moins 6 casiers. 
Variantes de molettes déjà connues: il s'agit :

d'une part, de molettes à casiers hachurés obliques qui se rapprochent des molettes d'Unverzagt et $\mathrm{G}$. Chenet 2, 189 (tessons Ar. 25, 26), et - pour autant que la taille des tessons permette d'en juger - des molettes 5, 6 (2 fois), 8 (tessons Ar. 29, 27 et 30,28 ), mais s'en différencient par de nombreux détails d'exécution ;

d'autre part, de molettes diverses qui se rapprochent beaucoup des nos 158 (carrés un peu plus petits) (Ar. 23), 233 (diverses irrégularités) (Ar. 32 a, b, c, appartenant probablement à deux vases différents), 252 (creux un peu plus petits) (Ar. 33), 347 (globules plus gros et hachures moins régulières) (Ar. 21).

Molettes déjà connues: 209, 268, 272 (Ar. 19, 20, 18), avec certitude; 62 ou var. 62 (Ar. 22) 157 ou var. 157 (Ar. 24 et 39), pour lesquelles l'exiguïté des tessons ne permet pas la certitude. La molette 268 est ici utilisée à l'envers, c'est-à-dire que les oves sont renversées par rapport à la représentation de G. Chenet.

Molelte complélée: la molette figurant sur le bol dont il a déjà été question (0. 487) est parfaitement identifiable avec la molette 212 de G. Chenet; toutefois celle-ci n'élait connue jusqu'ici que par le petit fragment publié par W. Unverzagt, alors que notre trouvaille en permet le relevé complet qui est donné $\dot{a}$ la figure 2 , sous le $n^{0} 18$ : molette à 9 casiers et un petit casier supplémentaire de liaison.

La villa des Terres Noires de GuiryGadancourt prend place parmi les gisements importants, actuellement connus, ayant fourni de la céramique d'Argonne du Iv $^{\mathrm{e}}$ siècle décorée à la molette. Avec ses 33 molettes différentes (en négligeant les incertaines), dont 16 inédites $(+1$ de provenance voisine), cet établissement modeste occupe dès maintenant, de ce point de vue particulier, une place comparable à celle de Nimègue ou Strasbourg.

Pierre-Henri MitaRd. 